\title{
The Glymphatic Influx and Slow Delta Waves in Sleep and Anesthesia
}

\author{
J. Shashi Kiran Reddy ${ }^{1}$ and Sisir Roy ${ }^{1}$
}

Investigation of the mechanisms and pathways of waste clearance in the brain has received much attention lately because of their functional significance and clinical implications $(1,2)$. In a recent study (3), Hablitz et al. made an attempt to bring us one step forward in tackling the puzzling question of how and when the brain clears its waste. Here, the authors studied the influence of different anesthetics (known to emulate sleep like conditions) on the glymphatic influx, which subsequently promotes waste clearance. It is the first study to demonstrate that the rate of glymphatic influx can be correlated to the prevalence of high EEG delta power and low heart rate variability. This relation also seems to vary uniquely based on the type of anesthetic substance administered. Analyzing the findings, they propose that the prevalence of the slow delta waves of highly synchronized neuronal activity promotes glymphatic influx. But we suggest, while assigning such role to the delta waves alone, findings may benefit if analyzed and interpreted in relation to a recent observation of the presence of regional delta oscillations in REM sleep; which hitherto is considered as the characteristic hallmark of non-REM sleep (4). Moreover, two clusters of delta waves with distinctive properties were also identified in both REM and non-REM sleep. Considering the variation in delta prevalence across different sleep phases, it becomes important to know to what extent the occurrence of the slow delta waves promotes glymphatic influx. So, the reported findings in (3) cannot be generalized directly to sleep. Therefore, the effect of different phases of sleep on glymphatic influx should be considered to investigate the role of delta waves in promoting waste clearance.

A recent study indicates that the lateral position of the body promotes efficient glymphatic transport compared to supine and prone positions (5), so one should also consider the impact of body posture on the glymphatic transport. Such findings become more relevant for research studies on sleep and anesthesia investigating the waste clearance mechanisms and pathways in the brain. While studying the effect of different anesthetic substances on the glymphatic influx, in (3), the role of intracranial pressure (ICP) has not been analyzed further. This is because, the authors report that they have not observed any significant changes in the ICP in relation to different anesthetics studied. But we want to emphasize that, in such case, findings in (3) cannot be applied to sleep condition directly, as ICP is characterized by significant changes during sleep (6). Overall, studying the variation in slow delta waves, body posture and intracranial pressure during different phases of sleep would help better in analyzing and interpreting the findings in (3).

\section{References:}

1. L. Xie et al., Science 342, 373-377 (2013).

\footnotetext{
${ }^{1}$ Consciousness Studies Programme, National Institute of Advanced Studies (NIAS), IISc Campus, Bengaluru, India. Email Address: jumpalreddy@live.com,jumpal_shashi@yahoo.com and sisir.sisirroy@gmail.com
} 
2. J. K. Holth et al., Science 363, 880 (2019).

3. L. M. Hablitz et al., Sci Adv 5(2) (2019).

4. G. Bernardi et al., J. Neurosci. 39 (14), 2686-2697 (2019).

5. H. Lee et al., J. Neurosci. 35(31), 11034-11044 (2015).

6. H. Stephensen et al., Neurosurgery 59, 326-332 (2006). 